\title{
A Comparison of Xenon Plasma FIB Technology with Conventional Gallium LMIS FIB: Imaging, Milling, and Gas-Assisted Applications
}

\author{
R. Young*, C. Rue*, S. Randolph*, C. Chandler*, G. Franz**, R. Schampers**, A. Klumpp***, and \\ L. Kwakman** \\ *FEI Company, 5350 NE Dawson Creek Drive, Hillsboro, OR 97124 \\ **FEI Company, Building AAE, PO Box 80066, 5600 KA Eindhoven, The Netherlands \\ ***Fraunhofer EMFT, Hansastrasse 27d, 80686 Munich, Germany
}

Focused ion beam (FIB) systems incorporating Xe inductively coupled plasma (ICP) ion sources have recently been introduced. Typical applications for the semiconductor industry have already been investigated, including: bulk silicon trenching, through-silicon via (TSV) cross sectioning, and package-level deprocessing [1-3]. In this work, we present a detailed comparison between Xe plasma FIB and conventional Ga liquid metal ion source (LMIS) tools. Specifically, we investigate imaging, milling, and gas-assisted operations including metal deposition and silicon etching.

The beam current range of the plasma FIB (few pA to $>1 \mu \mathrm{A}$ ) is approximately two orders of magnitude larger than a typical gallium LMIS FIB (1 pA to $20 \mathrm{nA})$. At low currents, the gallium FIB resolution is superior to the plasma FIB resolution, but at high currents the situation is reversed. Due to differences in the angular intensities of the two sources, the performance of the plasma FIB begins to outperform Ga-LMIS at currents above $20 \mathrm{nA}$. Milling removal rates for silicon are shown in Fig. 1. An example section through a filled copper TSV is shown in Fig.2, where the Xe beam has been used for depositing the protective layer, milling the section, and then forming the final image. From an imaging point of view, it can be seen that the secondary electron contrast in the $\mathrm{Xe}^{+}$image is similar to that expected with a $\mathrm{Ga}^{+}$beam, with insulating layers appearing dark (due to passive voltage contrast) and metals exhibiting strong grains contrast (due to ion channeling). Gas-assisted deposition and etching have also been investigated. $\mathrm{XeF}_{2}$-assisted silicon removal rates of $1.5 \times 10^{6}$ $\mu^{3} /$ min were measured with the Xe-plasma FIB, in contrast to the previous Ga-LMIS record of $2 \times 10^{5} \mu \mathrm{m}^{3} / \mathrm{min}$ [4]. The results for metal deposition are mixed. Volumetric deposition rates for $\mathrm{Pt}$ films were three times higher for the Xe-plasma FIB, but the resistivity of the Ga-deposited films was significantly lower. The differences in resistivity are attributed to the contributions of implanted primary ions and differences in the density and porosity of the deposited films.

Overall, these findings indicate that while many plasma-FIB operations are similar to their Ga-LMIS counterparts, there are also important differences (beyond the larger beam current range) to understand, and take into account, to get the best applications results (Figs. 3 and 4).

\section{References}

[1] S.M. Kellogg et al., Microsc. Microanal. 16 (Suppl 2) (2010) 222

[2] R.J. Young, EDFA Magazine, Vol 13(1) (2011) 12

[3] P. Ramm et al., Proc. IMAPS Device Packaging Conference (2011)

[4] C. Rue et al., ISTFA 2008: Proc. 34th Int. Symp. for Testing and Failure Analysis (2008) 141

[5] A part of the work has been performed in the project JEMSiP_3D, which is funded by the Public Authorities in France, Germany, Hungary, The Netherlands, Norway and Sweden, as well as by the ENIAC Joint Undertaking 

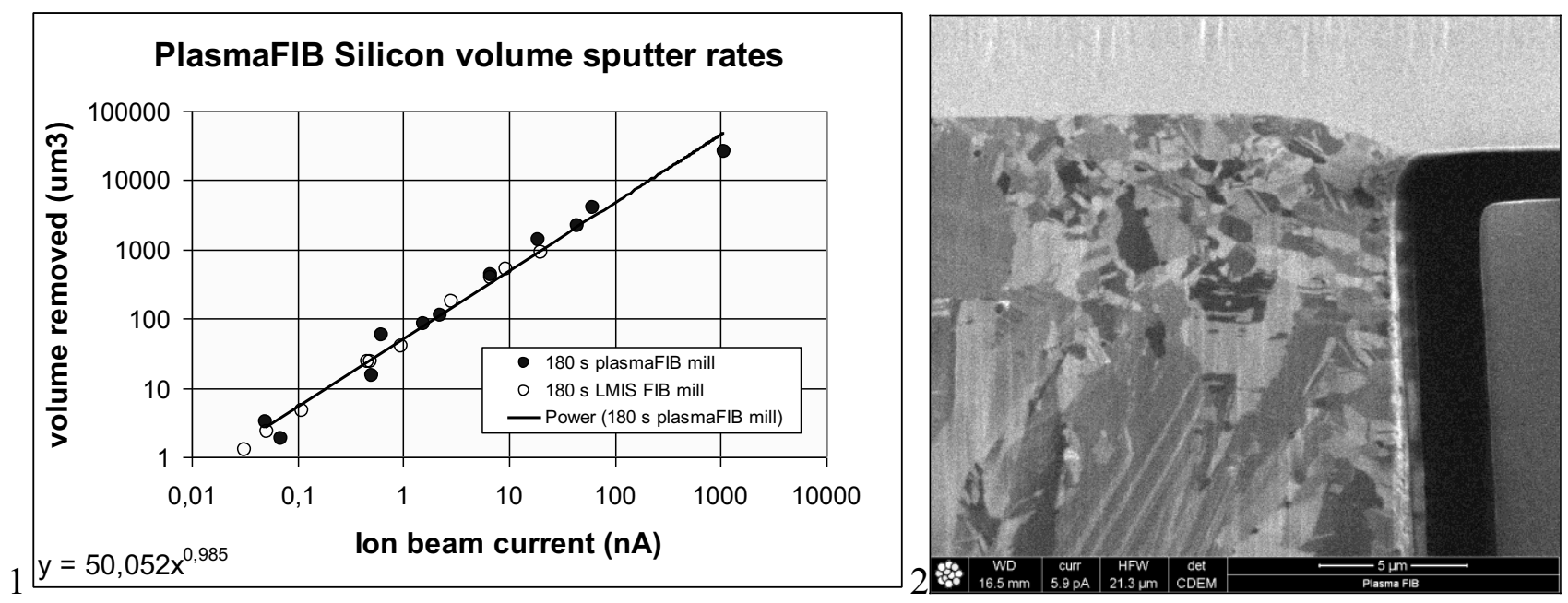

Fig. 1. Volume removal (in 180s) versus ion beam current for Xe-plasma and Ga-LMIS based FIB.

Fig 2. Corner of TSV structure sectioned and imaged by Xe plasma FIB, showing materials and grains contrast similar to that expected in a conventional Ga-FIB. Sample courtesy of Fraunhofer IWM Halle, prepared in the ENIAC ESIP project.
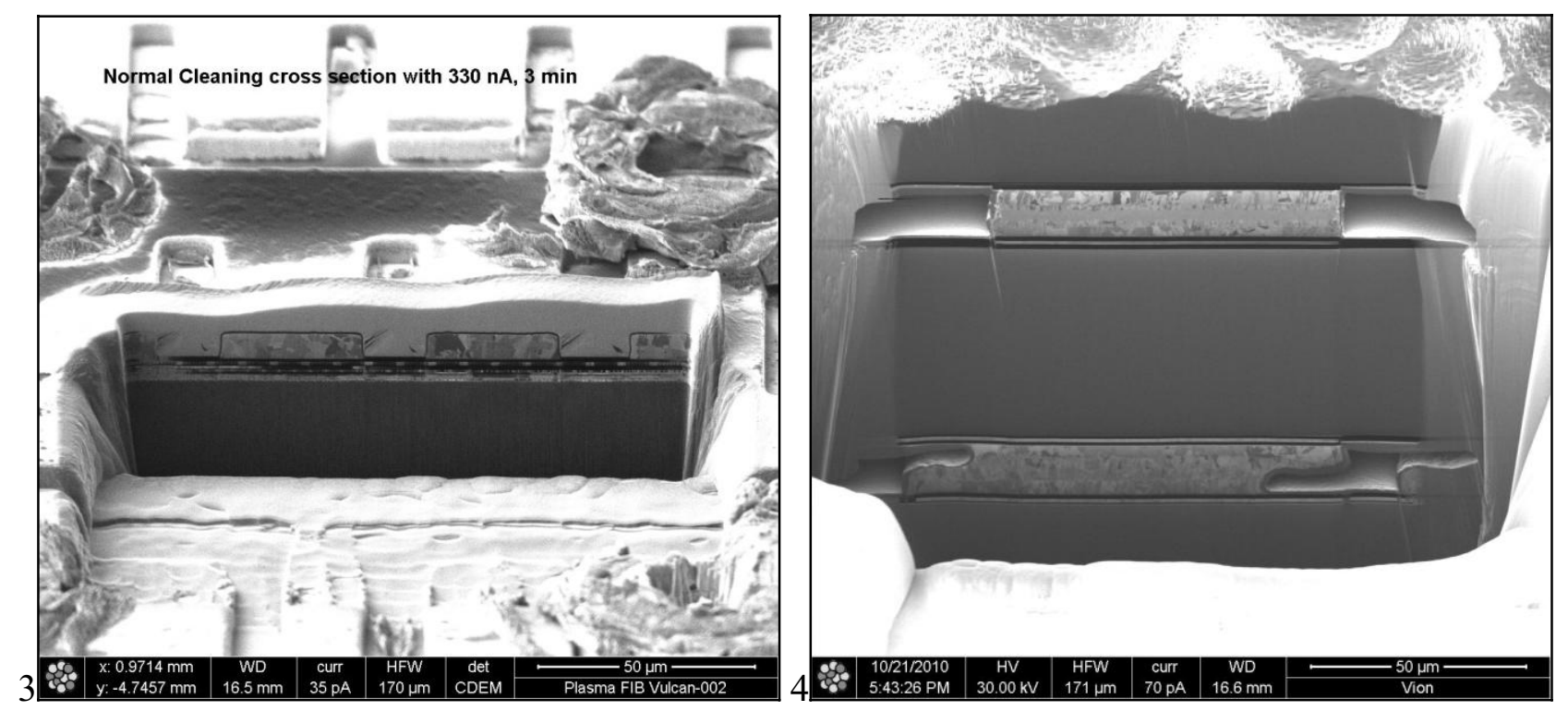

Fig. 3 Large cross section on an integrated circuit device prepared using the $\mathrm{Xe}^{+}$plasma FIB. A thick layer of Pt was deposited as a sacrificial cap prior to sectioning. The Pt thickness varies from 7 to $15 \mu \mathrm{m}$. Despite the enormous volume of metal deposited, the process required just minutes to complete.

Fig. 4. Cross section of a device with stacked die. $\mathrm{XeF}_{2}$-assisted silicon removal ("trenching") was used to remove the bulk of the overlying substrate silicon prior to sectioning. 